\title{
Über die Zeichen für die Planeten usw. auf Astrolabien.
}

In dem Werk von al Bêrânî ( $\dagger$ ca. 1048), Werk der Belehrung (Kitâb al Tafhim) ${ }^{1}$ ) über die Mathematik, Astronomie und Astrologie, das für die Ausdrücke dieser Wissenschaften Definitionen aufstellt, findet sich eine interessante Angabe über die Verwendung der Zeichen für die Sonne, den Mond und die Planeten auf den Astrolabien. Es heiBt dort: „Was sind die Zeichen, die sich auf der Oberfläche des Astrolabs befinden? Man schreibt auf sie nur die "Hadd“" ), die „Wagh“" $)$ und die „Mutallata“" $)$ der Gestirne. Der Raum ist zu eng, um die Namen der Gestirne einzuschreiben; man zeichnet sie mit byzantinischen Zeichen ein; diese sind in folgender Weise bei den Leuten der Kunst bekannt geworden."

\begin{tabular}{|c|c|c|c|c|c|c|}
\hline Jupiter & Saturn & Mars & Venus & Merkur & Sonne & Mond \\
\hline 1. ह & $Y$ & $\uparrow$ & $\uparrow$ & 1. $\&$ & \# & $\nabla$ \\
\hline 2. $t$ th & 专 & 平 & $q$ & 2. 7 & 8 & $\theta$ \\
\hline 3. 飞 & $K_{\varphi}$ & $\cos \hat{\phi}$ & 甲 $\Omega$ & 3. $\$ \gamma$ & $\phi$ & D oder $\mathbb{C}$ \\
\hline
\end{tabular}

Die erste Reihe enthält für die darüber stehenden Gestirne die Zeichen der Gestirne nach einer Berliner Handschrift des Tafhîm, die zweite nach einer Oxforder desselben Werkes; die letzteren war Herr Cowley so gütig mir mitzuteilen. Die dritte Reihe enthält die Kopps Palaeogr. crit. III S. 342 entnommenen und nacb Letronne (s. u.) ergänzten Zeichnungen, wie sie sich in abendländischen Handschriften finden.

Wir sehen aus al Bêrûnîs Angabe, daB auf dem Astrolab astrologische Wechselbezeichnungen eine Hauptrolle spielen. Nach den Mafâtîh haben die drei astrologischen Ausdrücke folgende Bedeutung (S. 215):

Al Mutallata ('Triplizitäten, 'Triquetra, Trigone). Je drei Zeichen des Tierkreises haben ein und dieselbe Natur, es gehört das Mutallata zu drei Gestirnen. Das eine ist der Herr (Sâhib) des Mutallata, der bei Tage vorangeht, der zweite geht bei Nacht voran und der dritte

1) Ich konnte die Berliner Handschrift Nr. 5665 benützen, Nr. 5666 enthält keine Abbildungen. Es existiert auch eine Oxforder Handschrift; vgl. Nicoll, Cat. Bibl. Bodl. II No. 282 p. 263.

2) $\mathrm{Zu}$ al Hadd (terme) vgl. Prolegomènes von Ibn Khaldún ed. Slane. Utbersetzung Notices et extraits des manuscrits etc. Bd. 20 S. 221, Anm. 1. Dort finden sich auch einige andere Angaben.

3) Zu al Wagh (face) vgl. auch Slane a. a. 0. Bd. 21 S. 180, Anm. 1.

4) $\mathrm{Zu}$ al Mutallața (Țrigones, Triplicites) vgl. auch Slane a. a. U. Bd. $\mathbf{0}$ S. 217, Anm. - Alle drei Ausdrücke bespricht auch Dozy in seinem Dictionnaire. 
ist ihr Genosse bei Tag und bei Nacht. So bilden der Widder, der Löwe und der Schütze ein Mutallata, es ist naB und trocken, seine Herrn (Rabb) sind bei Tage die Sonne, dann der Jupiter, bei Nacht der Jupiter und dann die Sonne, ihr Genosse bei Nacht und bei Tage der Saturn. - Stier, Aehre (Jungfrau) und Steinbock sind ein Mutallata, es ist kalt und trocken, seine Herrn sind bei Tage die Venus und der Mond, bei Nacht ist es umgekehrt, ihr Genosse ist der Mars. Zwillinge, Wage und Schöpfeimer (Wassermann) sind ein Mutallata, heiB und feucht, ihre Herrn sind am Tage der Jupiter und der Merkur, bei Nacht ist es umgekehrt, und ihr Genosse ist der Jupiter. Krebs, Skorpion und Fische sind ein Mutallata, kalt und feucht, seine Herrn sind bei Tage Venus und Mars, bei Nacht ist es umgekehrt, ihr Genosse ist der Mond. ${ }^{1}$ )

Al Wagh, al Sûra, al Darîgân²) und al Dahhg (Dekane). Ihre Bedeutung sind je $10^{\circ}$ eines jeden Tierkreiszeichens. Jedes Wagh hat einen Genossen (Șahị̂b) von den 7 Gestirnen.

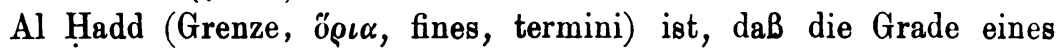
jeden Tierkreiszeichens zwischen die fünf Planeten verteilt sind, aber nicht in gleicher Weise. ${ }^{3}$ ) Jeder Teil heißt Hadd; im Persischen ist das Wort "Marz".

Aus der Angabe von al Bêrûnî geht hervor, daß auf den byzantinischen Astrolabien sich die Zeichen für Sonne, Mond und die Planeten fanden und zwar diejenigen, aus denen sich später die unsrigen entwickelt haben. Es dürfte dies eine interessante Ergänzung zu den Ausführungen von Letroune (Revu earchéol. III année, 1840, S. 258 ff.) sein, auf die Fr. Boll so gütig war mich hinzuweisen. Nach Letronne finden sich die Planetenzeichen weder auf Medaillen noch auf geschnittenen Steinen noch auf Basreliefs. Selbst die gnostischen Steine und Abraxas zeigen sie nicht. Dagegen sind sie in astrologischen und alchemistischen Traktaten verwendet; das älteste Manuskript, das Letronne kannte, geht auf das 10. Jahrhundert zurück, doch ist nach Fr. Boll diese Bezeichnungsart gewiB älter (irgendwelche systematische Durcharbeitung des Materials ist seit Letronne anscheinend nicht mehr vorgenommen worden).

Erlangen.

\section{Eilhard Wiedemann.}

1) Ctber diese Trigona und ihre Herrn, eine bekannte Lehre der griechischen Astrologie, vgl. Bouché-Leclercq, L'astrologie gr. p. 200 ff.; über die $\mathbf{Z}_{\rho \iota \alpha}$ ebd. $206 \mathrm{ff}$.

2) Darîgân ist ein pereisches Wort.

3) D. h. die Zahl der Grade ist nicht für jeden der 5 Planeten die gleiche. 